\title{
Petersen plane arrangements and a surface with multiple 7-secants
}

\author{
Hirotachi Abo, Holger P. Kley*and Chris Peterson* \\ (Communicated by R. Miranda)
}

\begin{abstract}
We study configurations of 2-planes in $\mathbb{P}^{4}$ that are combinatorially described by the Petersen graph. We discuss conditions for configurations to be locally Cohen-Macaulay and describe the Hilbert scheme of such arrangements. An analysis of the homogeneous ideals of these configurations leads, via linkage, to a class of smooth, general type surfaces in $\mathbb{P}^{4}$. We compute their numerical invariants and show that they have the unusual property that they admit (multiple) 7-secants. Finally, we demonstrate that the construction applied to Petersen arrangements with additional symmetry leads to surfaces with exceptional automorphism groups.
\end{abstract}

Key words. Plane arrangements, Petersen graph, automorphisms, 7-secants, general type surface, liaison, group actions, linkage.

2000 Mathematics Subject Classification. 14N20, 14J50, 14J25, 14M06, 05E20, 14 J10

\section{Introduction}

Linkage (or liaison) theory can provide a bridge between combinatorially interesting varieties such as $\ell$-plane arrangements (equidimensional unions of projective subspaces of $\mathbb{P}^{n}$ ) and geometrically interesting ones: smooth projective varieties. This paper studies one such bridge, connecting the class of 2-plane arrangements in $\mathbb{P}^{4}$ whose incidence structure may be described by the Petersen graph with a previously unpublished class of smooth surfaces.

We begin, in Section 2, with a review of the relevant combinatorics of the Petersen graph. Next, in Section 3, we associate a graph to an arrangement: the vertices are the linear spaces in the arrangement and two vertices are joined by an edge if the spaces meet in greater than the expected dimension. Our study of the geometry of 2-plane arrangements begins with a necessary combinatorial condition (Corollary 3.10) on the associated graph to insure that the underlying arrangement is locally Cohen-Macaulay (ICM). Since

\footnotetext{
${ }^{*}$ The second and third author were partially supported by NSF grant MSPA-MCS-0434351.
} 
linkage preserves the $1 \mathrm{CM}$ property, such a result is essential if we are interested in linking to smooth surfaces.

The discussion leads naturally to the consideration of 2-plane arrangements whose associated graph is the Petersen graph, which begs the following questions: do such arrangements exist, and are they (generally) locally Cohen-Macaulay? In Section 4, we answer both questions in the affirmative (Propositions 4.9) via an explicit and combinatorially interesting construction and show (Proposition 4.10) that in fact, all ICM Petersen arrangements arise in this manner. The modular nature of the construction has immediate benefits. For example, we are able to give detailed information (Theorem 4.11) about the Hilbert scheme of such arrangements.

Furthermore, the explicit construction allows a detailed description of the homogeneous ideal of an ICM Petersen arrangement; this is the subject of Section 5. In particular, we find (Theorem 5.7) that the ideal always contains six quintic forms. Thus, it is not surprising that Petersen arrangements may be linked, via a general pair of quintics, to (smooth) degree fifteen surfaces in $\mathbb{P}^{4}$. Ellingsrud and Peskine [8] proved that there exists a constant $d_{0}$ such that every smooth surface in $\mathbb{P}^{4}$ of degree $d>d_{0}$ is of general type. While the smallest possible value for $d_{0}$ is still an open problem, many believe that the answer is $d_{0}=15$. In any case, for surfaces in $\mathbb{P}^{4}$ there seems to be a propensity towards special behavior at this degree. For instance, the lowest degree of a non-arithmetically Cohen-Macaulay smooth quasi-complete intersection surface in $\mathbb{P}^{4}$ occurs in degree 15 . It is a surface of non-general type and can be used to construct the Horrocks-Mumford bundle on $\mathbb{P}^{4}$. Through linkage (or by considering the zero locus of a section of the Horrocks-Mumford bundle), we obtain another very special object; the abelian surface of degree 10. Furthermore, the largest known degree of an abelian surface and of a bielliptic surface occurs in degree 15 . This confluence of special behavior provided motivation to the authors to search in degree 15 (and degree 10) for other interesting phenomena. It should be noted that Ranestad and Aure (independently and unpublished) explored such a construction while considering the classification of low-degree surfaces in $\mathbb{P}^{4}$.

The degree fifteen surfaces, constructed through linkage from ICM Petersen arrangements, are the subject of Section 6. In particular, we show that they are smooth and of general type (Theorem 6.2). By exploiting the link between combinatorics and geometry once more, we show (Theorem 6.6) that the surfaces so obtained have the surprising property that they admit a one-dimensional family of 6-secant lines and fifteen 7-secant lines. This is unusual in that surfaces in $\mathbb{P}^{4}$ typically have a finite number of 6-secants and no 7 -secants. In particular, we know of no other examples of surfaces in $\mathbb{P}^{4}$, of such low degree, that have as many 7-secants. Finally, in Theorem 7.1, we exploit the family of 2-planes as a combinatorial object, determine configurations with non-trivial automorphism groups, and show how to use such configurations to generate examples of smooth surfaces with non-trivial automorphism groups.

Note. All computer algebra scripts used in examples and computations throughout the present article may be found at [1].

Notation. For simplicity, fix an algebraically closed field $K$ of characteristic zero. (In fact, the reader will note that all results will hold over sufficiently large algebraic extensions of the prime field in all but finitely many positive characteristics.) 
For $n$ a non-negative integer, let $\mathbb{P}^{n}:=\mathbb{P}\left(K^{n+1}\right)$ denote the projective space of dimension $n$ over $K$. By a linear (sub)space (of $\mathbb{P}^{n}$ ) we mean $\mathbb{P}(V)$ for some vector subspace $V \subset K^{n+1}$. If $\operatorname{dim} V=\ell+1$, we also refer to $\mathbb{P}(V)$ as an $\ell$-plane in $\mathbb{P}^{n}$. Finally, for linear subspaces $\Lambda_{1}=\mathbb{P}(V)$ and $\Lambda_{2}=\mathbb{P}(W)$ of $\mathbb{P}^{n}$, let $\overline{\Lambda_{1}, \Lambda_{2}}=\mathbb{P}(V+W)$ denote the linear space they span.

Acknowledgments. The authors would like to thank Rick Miranda, Kristian Ranestad, Bernd Sturmfels and Ravi Vakil for stimulating conversations and questions.

\section{The Petersen graph}

In this section, we recall a few definitions from graph theory. We then define the Petersen graph and outline some of its standard properties.

Definition 2.1. Let $\Gamma$ be a graph with vertex set $V$ and edge set $E$.

- A matching in $\Gamma$ is a subset $E^{\prime} \subset E$ such that no two edges in $E^{\prime}$ share a vertex.

- A perfect matching in $\Gamma$ is a matching that covers every vertex in $\Gamma$.

- An independent set of vertices of $\Gamma$ is a subset $S \subset V$ such that no pair of vertices in $S$ form an edge of $\Gamma$.

- A maximal independent set of vertices is an independent set of vertices which is not properly contained in any other independent set of vertices.

- The girth of $\Gamma$ is the length of a shortest, non-trivial cycle in $\Gamma$. If $\Gamma$ contains no non-trivial cycles, it is said to have infinite girth.

By a slight abuse of notation, we write $\{0,1,2,3,4\}=\mathbb{Z} /(5)$ for (the elements of) the integers modulo 5 .

The Petersen graph is the graph $\Gamma_{\text {Pete }}$ with vertex set

$$
V\left(\Gamma_{\text {Pete }}\right):=\left\{0,1,2,3,4,0^{\prime}, 1^{\prime}, 2^{\prime}, 3^{\prime}, 4^{\prime}\right\}=\left\{i, i^{\prime}: i \in \mathbb{Z} /(5)\right\}
$$

and edge set

$$
\begin{aligned}
E\left(\Gamma_{\text {Pete }}\right): & =\left\{01,12,23,34,40,00^{\prime}, 11^{\prime}, 22^{\prime}, 33^{\prime}, 44^{\prime}, 0^{\prime} 2^{\prime}, 1^{\prime} 3^{\prime}, 2^{\prime} 4^{\prime}, 3^{\prime} 0^{\prime}, 4^{\prime} 1^{\prime}\right\} \\
& =\left\{i(i+1), i i^{\prime}, i^{\prime}(i+2)^{\prime}: i \in \mathbb{Z} /(5)\right\} .
\end{aligned}
$$

One standard way to realize $\Gamma_{\text {Pete }}$ is shown in Figure 1. Notice that $\Gamma_{\text {Pete }}$ has diameter 2 and girth 5 .

Henceforth, let $\rho \in \operatorname{Aut}\left(\Gamma_{\text {Pete }}\right)$ be the "rotation by $2 \pi / 5$," i.e., the automorphism whose action on $V\left(\Gamma_{\text {Pete }}\right)$ is given by

$$
\rho(i)=i+1 \text { and } \rho\left(i^{\prime}\right)=(i+1)^{\prime} \text { for } i \in \mathbb{Z} /(5),
$$

let $\tau \in \operatorname{Aut}\left(\Gamma_{\text {Pete }}\right)$ be the automorphism whose action on $V\left(\Gamma_{\text {Pete }}\right)$ is given (in cycle notation) by

$$
\tau:=\left(00^{\prime}\right)\left(13^{\prime} 42^{\prime}\right)\left(21^{\prime} 34^{\prime}\right)
$$




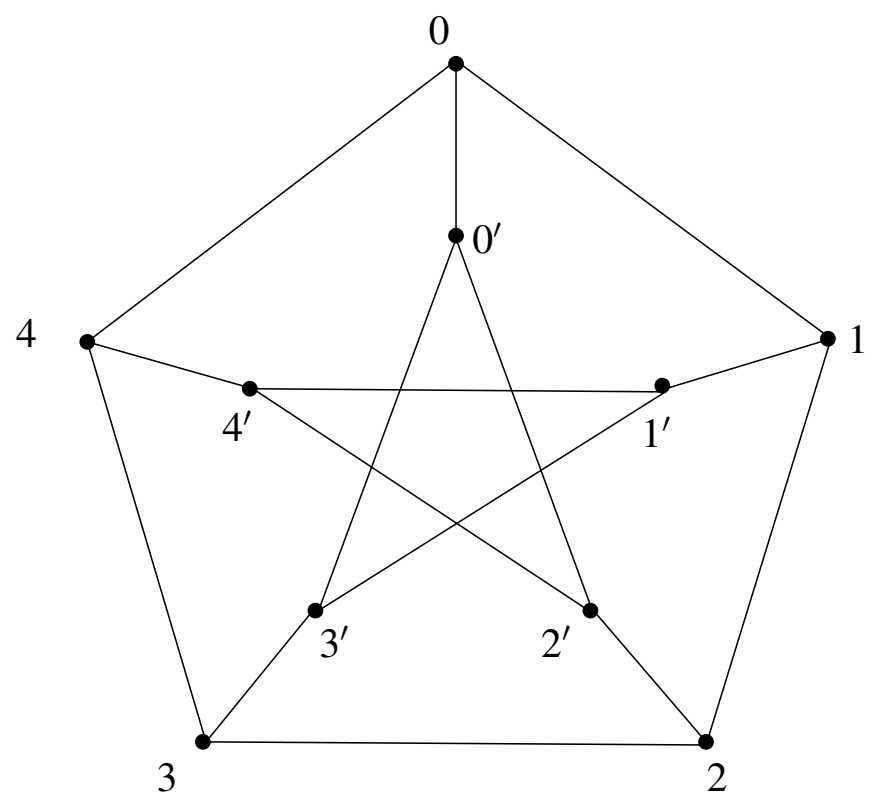

Figure 1. The Petersen graph.

and let $\omega \in \operatorname{Aut}\left(\Gamma_{\text {Pete }}\right)$ be the automorphism whose action on $V\left(\Gamma_{\text {Pete }}\right)$ is given (in cycle notation) by

$$
\omega:=\left(21^{\prime}\right)\left(34^{\prime}\right)\left(2^{\prime} 3^{\prime}\right) .
$$

Referring to Figure 1, $\tau$ more or less "turns $\Gamma_{\text {Pete }}$ inside-out." Also, observe that

$$
\sigma:=\tau^{2}=(14)(23)\left(1^{\prime} 4^{\prime}\right)\left(2^{\prime} 3^{\prime}\right) \in \operatorname{Aut}\left(\Gamma_{\text {Pete }}\right)
$$

is the "reflection across the vertical."

We identify the maximal independent vertex set

$$
V_{0}:=\left\{1,4,2^{\prime}, 3^{\prime}\right\} \subset V\left(\Gamma_{\text {Pete }}\right)
$$

of $\Gamma_{\text {Pete }}$ and observe that any 4-element maximal independent vertex set of $\Gamma_{\text {Pete }}$ is one of

$$
V_{j}:=\rho^{j} V_{0}
$$

for $j=0, \ldots, 4$. Notice that each vertex of $\Gamma_{\text {Pete }}$ lies in precisely two of the $V_{j}$ and that any two of the $V_{j}$ intersect in exactly one vertex of $\Gamma_{\text {Pete }}$. Consequently, Aut $\left(\Gamma_{\text {Pete }}\right)$ acts faithfully on $\left\{V_{0}, \ldots, V_{4}\right\}$. Furthermore, $\rho$ acts as a 5-cycle on $\left\{V_{0}, \ldots, V_{4}\right\}$ while $\omega$ acts as the transposition $\left(V_{1} V_{4}\right)$ on $\left\{V_{0}, \ldots, V_{4}\right\}$, so we recover the well-known

Proposition 2.2. The automorphism group of the Petersen graph is isomorphic to the symmetric group on 5 letters. 
Similarly, we identify the perfect matching

$$
E_{5}:=\left\{00^{\prime}, 11^{\prime}, 22^{\prime}, 33^{\prime}, 44^{\prime}\right\} \subset E\left(\Gamma_{\text {Pete }}\right),
$$

and observe that every perfect matching of $\Gamma_{\text {Pete }}$ is either $E_{5}$ or one of

$$
E_{j}:=\rho^{j} \omega E_{5} \quad \text { for } j=0, \ldots, 4 .
$$

Clearly, Aut $\left(\Gamma_{\text {Pete }}\right)$ acts transitively on $\left\{E_{0}, \ldots, E_{5}\right\}$, so the group

$$
G:=\operatorname{Stab}\left(E_{5}\right) \leq \operatorname{Aut}\left(\Gamma_{\text {Pete }}\right)
$$

has order 20. One easily checks that $\rho, \tau \in G$, and concludes that

$$
G=\langle\rho, \tau\rangle \leq \operatorname{Aut}\left(\Gamma_{\text {Pete }}\right) .
$$

Finally, we identify

$$
D:=\langle\rho, \sigma\rangle=\left\langle\rho, \tau^{2}\right\rangle \leq G,
$$

which is isomorphic to the dihedral group of order 10.

\section{Arrangements and their incidence graphs}

In this section we define our basic objects of study and prove a simple but fundamental proposition about their algebro-geometric structure.

We begin with a trivial but useful observation about linear spaces:

Remark 3.1. Let $P, Q$ and $R \subset \mathbb{P}^{n}$ be linear spaces. If

$$
\operatorname{dim}(P \cap R)+\operatorname{dim}(Q \cap R)-\operatorname{dim} R \geq \operatorname{dim}(P \cap Q),
$$

then $(P \cap Q) \subset R$. In particular, if $R$ is a 2-plane meeting $P$ and $Q$ in lines and if $P \cap Q=\{p\}$, then $p \in R$.

Definition 3.2. For positive integers $\ell<n$, an $\ell$-plane arrangement in $\mathbb{P}^{n}$ is a finite collection of $\ell$-dimensional linear subspaces of $\mathbb{P}^{n}$.

Notation 3.3. Let $\mathcal{A}$ be an $\ell$-plane arrangement in $\mathbb{P}^{n}$, let $S=K\left[x_{0}, \ldots, x_{n}\right]$ be the homogeneous coordinate ring of $\mathbb{P}^{n}$, let $H \subset \mathbb{P}^{n}$ be a hyperplane, and let $p \in \mathbb{P}^{n}$. Then we write

- $V_{\mathcal{A}}:=\bigcup_{\Lambda \in \mathcal{A}} \Lambda$ for the corresponding projective variety in $\mathbb{P}^{n}$,

- $I_{\mathcal{A}} \subset S$ for the homogeneous ideal of $V_{\mathcal{A}}$ and $\mathcal{I}_{\mathcal{A}}$ for its ideal sheaf in $\mathcal{O}_{\mathbb{P}^{n}}$,

- $\mathcal{A}_{p}:=\{\Lambda \in \mathcal{A}: p \in \Lambda\}$ for the subarrangement of $\ell$-planes in $\mathcal{A}$ which pass through $p$,

- $H \cap \mathcal{A}:=\{H \cap \Lambda: \Lambda \in \mathcal{A}\}$ for the hyperplane section of $\mathcal{A}$ by $H$, and

- $\overline{p, \mathcal{A}}:=\{\overline{p, \Lambda}: \Lambda \in \mathcal{A}\}$ for the cone over $\mathcal{A}$ with vertex $p$. 
Remark 3.4. We freely use three simple facts about cones and hyperplane sections of $\ell$-plane arrangements:

- If $\mathcal{A}$ is an $\ell$-plane arrangement in $\mathbb{P}^{n}$ and if $H \subset \mathbb{P}^{n}$ is a hyperplane not containing any of the members of $\mathcal{A}$, then $H \cap \mathcal{A}$ is an $(\ell-1)$-plane arrangement in $H$.

- If $\mathcal{A}$ is an $(\ell-1)$-plane arrangement in a hyperplane $H \subset \mathbb{P}^{n}$ and if $p \notin H$, then $\overline{p, \mathcal{A}}$ is an $\ell$-plane arrangement in $\mathbb{P}^{n}$.

- For $p \in \mathbb{P}^{n}$ and $H \subset \mathbb{P}^{n}$ a general hyperplane, $\mathcal{A}_{p}=\overline{p, H \cap \mathcal{A}_{p}}$.

In general, we will say that an arrangement has a certain geometric property if its corresponding variety does. In particular, we have:

Definition 3.5. Let $\mathcal{A}$ be an $\ell$-plane arrangement in $\mathbb{P}^{n}$ and let $p \in V_{\mathcal{A}}$ be a point corresponding to the prime ideal $\mathfrak{p} \subset S$. We say that $\mathcal{A}$ is arithmetically Cohen-Macaulay $(a C M)$ if the homogeneous coordinate ring $S / I_{\mathcal{A}}$ is a Cohen-Macaulay ring. We say that $\mathcal{A}$ is locally Cohen-Macaulay $(I C M)$ at $p$ if the local ring $\left(S / I_{\mathcal{A}}\right)_{\mathfrak{p}}$ is Cohen-Macaulay. Finally, we say that $\mathcal{A}$ is locally Cohen-Macaulay if it is ICM at all closed points $p \in V_{\mathcal{A}}$.

The following proposition is useful in understanding when an arrangement is $1 \mathrm{CM}$ at a point.

Proposition 3.6. Let $\mathcal{A}$ be an $\ell$-plane arrangement in $\mathbb{P}^{n}$ with $\ell \geq 2$. Let $p \in V_{\mathcal{A}}$. Let $H$ be a hyperplane not containing any plane of $\mathcal{A}$. The following are equivalent:

(i) $\mathcal{A}$ is lCM at $p$.

(ii) $\mathcal{A}_{p}$ is lCM at $p$.

(iii) $\mathcal{A}_{p}$ is $a C M$.

(iv) $H \cap \mathcal{A}_{p}$ is $a C M$.

Proof. The equivalence of (i) and (ii) is clear. The equivalence of (iii) and (iv) is an immediate corollary of a result of Huneke and Ulrich [6]. Finally, the equivalence of (ii) and (iv) follows because $\mathcal{A}_{p}$ is a cone over $H \cap \mathcal{A}_{p}$, and a variety is aCM if and only if the cone over the variety is $1 \mathrm{CM}$ at the vertex of the cone.

Example 3.7. Consider distinct 2-planes $P_{1}, P_{2}, P_{3}, P_{4} \subset \mathbb{P}^{4}$ not all contained in a hyperplane. Suppose that $P_{1} \cap P_{3}=P_{2} \cap P_{4}=P_{1} \cap P_{4}=\{p\}$ consists of a single point while $P_{1} \cap P_{2}, P_{2} \cap P_{3}$, and $P_{3} \cap P_{4}$ are lines (necessarily passing through $p$ by Remark 3.1). Then the arrangement $\left\{P_{1}, P_{2}, P_{3}, P_{4}\right\}$ is a cone over a projection into $\mathbb{P}^{3}$ of a degenerate rational normal curve of degree 4 . Such curves are not aCM and hence the arrangement $\left\{P_{1}, P_{2}, P_{3}, P_{4}\right\}$ is not lCM.

It is useful to keep track of those pairs of planes in an $\ell$-plane arrangement that are in special position with respect to each other. We represent this data via a graph.

Definition 3.8. If $\mathcal{A}$ is an $\ell$-plane arrangement in $\mathbb{P}^{n}$, the incidence graph of $\mathcal{A}$ is the graph $\Gamma(\mathcal{A})$, with vertices the planes of $\mathcal{A}$ and edges the pairs of planes with special intersection. In other words, $\Gamma(\mathcal{A})$ has vertex set $\mathcal{A}$, and edge set $\left\{\Lambda \Lambda^{\prime}: \operatorname{dim} \Lambda \cap \Lambda^{\prime}>\right.$ $n-2 \ell\}$. 
Remark 3.9. Because it captures non-generic behavior only between pairs of $\ell$-planes, the graph $\Gamma(\mathcal{A})$ may not completely characterize the geometry of the arrangement $\mathcal{A}$. For example, in $\mathbb{P}^{4}$, both the arrangement of three 2-planes all containing a common line and the arrangement of three 2-planes meeting pairwise in three distinct lines have the complete graph on three vertices as their incidence graphs. (These arrangements could, however, be distinguished by an incidence complex.)

Corollary 3.10. Let $\mathcal{A}$ be a 2-plane arrangement in $\mathbb{P}^{4}$ and let $p \in V_{\mathcal{A}}$. If $\Gamma\left(\mathcal{A}_{p}\right)$ is disconnected, then $\mathcal{A}$ is not lCM at $p$. As a partial converse, if $\Gamma\left(\mathcal{A}_{p}\right)$ is connected and of order at most three, then $\mathcal{A}$ is lCM at $p$.

Proof. Let $H$ be a hyperplane not passing through $p$. Then $\mathcal{A}_{p}$ is a cone over the arrangement $\mathcal{L}=\mathcal{A}_{p} \cap H$ of lines in $H=\mathbb{P}^{3}$, and by Proposition 3.6, $\mathcal{A}$ is 1CM at $p$ if and only if $\mathcal{L}$ is aCM. Now $\Gamma\left(\mathcal{A}_{p}\right)$ is connected if and only if $V_{\mathcal{L}}$ is connected, and disconnected curves are not aCM. Conversely, suppose $V_{\mathcal{L}}$ is connected and has at most three components. Then $V_{\mathcal{L}}$ is either a complete intersection, a cone over three non-collinear points in a plane, or a degenerate twisted cubic, all of which are aCM.

Remark 3.11. Let $\mathcal{A}$ be a 2-plane arrangement in $\mathbb{P}^{4}$. Corollary 3.10 suggests that we should expect $\mathcal{A}$ to be $\mathrm{ICM}$ if $\Gamma(\mathcal{A})$ is connected and of diameter at most 2 . On the other hand, Example 3.7 suggests that if the diameter of $\Gamma(\mathcal{A})$ exceeds 2 , then $\mathcal{A}$ may well fail to be $\mathrm{lCM}$. If $\Gamma(\mathcal{A})$ is disconnected, $\mathcal{A}$ fails to be $1 \mathrm{CM}$ in all cases.

\section{Petersen arrangements}

For the remainder of this paper, a plane means a 2-plane. In Remark 3.11, we observed that a plane arrangement $\mathcal{A}$ in $\mathbb{P}^{4}$, constructed in such a way that $\Gamma(\mathcal{A})$ has diameter 2 , is expected to be $1 \mathrm{CM}$. Among the graphs of finite girth, only those of girth $\leq 5$ may have diameter $\leq 2$. Thus, graphs of girth 5 and diameter 2 are a natural place to look for interesting examples of arrangements.

The simplest such graph is the pentagon, and one can readily construct a plane arrangement $\mathcal{A}$ in $\mathbb{P}^{4}$ such that $\Gamma(\mathcal{A})$ is isomorphic to the pentagon. This plane arrangement appears as a degenerate case in the family containing the elliptic quintic scrolls. Here elliptic quintic scrolls are ruled surfaces over elliptic curves embedded in $\mathbb{P}^{4}$ in such a way that all the fibers have degree 1 . These surfaces determine one of the few known families of smooth irregular surfaces in $\mathbb{P}^{4}$. The (degenerate) elliptic quintic scroll $V_{\mathcal{A}}$ is cut out by five cubic hypersurfaces and can be linked in the complete intersection of two such cubic hypersurfaces to the Veronese surface (which is known as the only smooth non-degenerate surface in $\mathbb{P}^{4}$ that is not linearly normal).

As we observed in Section 2, the Petersen graph $\Gamma_{\text {Pete }}$ likewise has diameter 2 and girth 5 .

Definition 4.1. A (two-)plane arrangement $\mathcal{A}$ in $\mathbb{P}^{4}$ is a Petersen arrangement if $\Gamma(\mathcal{A}) \simeq$

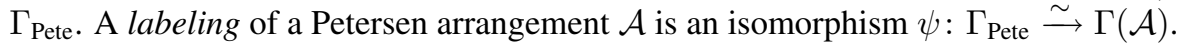


Several natural questions are: Do Petersen arrangements exist? If so, how big is the family of such arrangements? Do 1CM Petersen arrangements exist? If so, can liaison theory be used to produce (smooth) surfaces from Petersen arrangements? Do Petersen arrangements with extra structure (such as a prescribed automorphism group) exist? The remainder of this article addresses these questions.

4.1 Construction of Petersen arrangements. Let $\mathcal{L}=\left\{L_{i}\right\}_{i \in \mathbb{Z} /(5)}$ be an indexed collection of lines in $\mathbb{P}^{4}$. Suppose that $\mathcal{L}$ satisfies

Condition 1. $L_{0}, \ldots, L_{4}$ are pairwise skew.

Then for each pair $\{i, j\}$ of distinct elements of $\mathbb{Z} /(5), L_{i}$ and $L_{j}$ span a hyperplane $H_{i, j}:=\overline{L_{i}, L_{j}}$. Clearly, $H_{i, j}=H_{j, i}$.

Suppose that $\mathcal{L}$ also satisfies

Condition 2. No three lines of $\mathcal{L}$ are contained in a hyperplane.

Lemma 4.2. The following are equivalent conditions on a collection $\mathcal{L}$ satisfying Condition 1:

(i) Condition 2.

(ii) For all choices of distinct $i, j, k \in \mathbb{Z} /(5)$, the hyperplanes $H_{i, j}$ and $H_{i, k}$ are distinct.

(iii) For all choices of distinct $i, j, k \in \mathbb{Z} /(5)$, there is a unique "trisecant" line $M_{i, j, k}$ meeting $L_{i}, L_{j}$ and $L_{k}$.

(iv) For all choices of three distinct $i, j, k \in \mathbb{Z} /(5)$, there is a unique plane containing $L_{i}$ which meets $L_{j}$ and $L_{k}$.

Proof. This is immediate. The key observations are that

$$
M_{i, j, k}=H_{i, j} \cap H_{j, k} \cap H_{k, i},
$$

and that

$$
H_{i, j} \cap H_{i, k}=\overline{L_{i}, M_{i, j, k}}
$$

is the unique plane containing $L_{i}$ and meeting $L_{j}$ and $L_{k}$.

For each $i \in \mathbb{Z} /(5)$, we define two planes

$$
P_{i}:=H_{i, i+1} \cap H_{i, i-1} \quad \text { and } \quad P_{i}^{\prime}:=H_{i, i+2} \cap H_{i, i-2}
$$

and set

$$
\mathcal{A}=\mathcal{A}(\mathcal{L}):=\left\{P_{i}, P_{i}^{\prime}: i \in \mathbb{Z} /(5)\right\} .
$$

We would like to show that $\mathcal{A}$ is a Petersen arrangement. There are, however, two potential problems: $\Gamma(\mathcal{A})$ could contain $\Gamma_{\text {Pete }}$ as a proper subgraph, and some of the planes in $\mathcal{A}$ could coincide. To eliminate these possibilities, we require that $\mathcal{L}$ additionally satisfies

Condition 3. No plane containing a line of $\mathcal{L}$ meets three other lines of $\mathcal{L}$. 
Lemma 4.3. The following are equivalent conditions on a collection $\mathcal{L}$ satisfying Conditions 1 and 2:

(i) Condition 3.

(ii) $L_{\ell} \cap \overline{L_{i}, M_{i, j, k}}=\emptyset$ for all choices of distinct $i, j, k, \ell \in \mathbb{Z} /(5)$.

(iii) $H_{i, j} \cap H_{i, k} \cap H_{i, \ell}=L_{i}$ for all choices of distinct $i, j, k, \ell \in \mathbb{Z} /(5)$.

(iv) $M_{i, j, k} \cap M_{i, j, \ell}=\emptyset$ for all choices of distinct $i, j, k, \ell \in \mathbb{Z} /(5)$.

Proof. This is immediate in light of Lemma 4.2.

We can now establish

Proposition 4.4. Let $\mathcal{L}=\left\{L_{i}\right\}_{i \in \mathbb{Z} /(5)}$ be an indexed collection of lines in $\mathbb{P}^{4}$ satisfying Conditions 1-3. Then $\mathcal{A}(\mathcal{L})$ is a Petersen arrangement with labeling $\psi_{\mathcal{L}}: \Gamma_{\text {Pete }} \rightarrow \Gamma(\mathcal{A})$ given by $\psi_{\mathcal{L}}(i)=P_{i}$ and $\psi_{\mathcal{L}}\left(i^{\prime}\right)=P_{i}^{\prime}$. Moreover, $P_{i} \cap P_{i}^{\prime}=L_{i}$.

Proof. Since $\psi_{\mathcal{L}}$ is a bijection on vertices, it suffices to show that it induces a bijection of edges, which we establish as a series of claims. Let $i \in \mathbb{Z} /(5)$.

Claim. $\operatorname{dim}\left(P_{i} \cap P_{i+1}\right)=1$.

Proof. By construction, $P_{i} \cap P_{i+1}=H_{i-1, i} \cap H_{i, i+1} \cap H_{i+1, i+2}$, which is as least one-dimensional. On the other hand, $P_{i}$ contains $L_{i}$ and $P_{i+1}$ contains $L_{i+1}$ and since these lines are skew by Condition $1, P_{i} \neq P_{i+1}$.

Claim. $\operatorname{dim}\left(P_{i}^{\prime} \cap P_{i+2}^{\prime}\right)=1$.

Proof. This is essentially identical to the previous case.

Claim. $\operatorname{dim}\left(P_{i} \cap P_{i+2}\right)=0$.

Proof. Combining (4.2) with (ii) of Lemma 4.3 shows that $P_{i}=\overline{L_{i}, M_{i-1, i, i+1}}$ does not meet $L_{i+2} \subset P_{i+2}$. It follows that $P_{i}$ and $P_{i+2}$ cannot meet in a line.

Claim. $\operatorname{dim}\left(P_{i}^{\prime} \cap P_{i+1}^{\prime}\right)=0$.

Proof. This is essentially identical to the previous case.

Claim. $P_{i} \cap P_{i}^{\prime}=L_{i}$.

Proof. Using (iii) of Lemma 4.3,

$$
P_{i} \cap P_{i}^{\prime}=H_{i-1, i} \cap H_{i, i+1} \cap H_{i-2, i} \cap H_{i, i+2}=L_{i} \cap H_{i, i+2}=L_{i} .
$$

Claim. $\operatorname{dim}\left(P_{i} \cap P_{j}^{\prime}\right)=0$ for all $j \neq i$.

Proof. We consider two cases: $P_{i} \cap P_{i+1}^{\prime}$ and $P_{i} \cap P_{i+2}^{\prime}$. The other two cases are essentially identical. Keeping in mind that indices are calculated modulo five, we have

$$
P_{i} \cap P_{i+1}^{\prime}=H_{i-1, i} \cap H_{i, i+1} \cap H_{i-1, i+1} \cap H_{i+1, i-2} .
$$

Notice that three terms involve the index $i+1$. Applying (iii) of Lemma 4.3, we reduce to

$$
P_{i} \cap P_{i+1}^{\prime}=H_{i-1, i} \cap L_{i+1}
$$

which must be a point by Condition 2 .

For the second case, an analogous argument shows that

$$
P_{i} \cap P_{i+2}^{\prime}=H_{i-1, i} \cap H_{i, i+1} \cap H_{i, i+2} \cap H_{i+2, i-1}=H_{i+2, i} \cap L_{i}
$$

is also a point. 
Remark 4.5. The symmetric group $\mathfrak{S}=\mathfrak{S}_{\mathbb{Z} /(5)}$ acts naturally on the indexing of a collection $\mathcal{L}=\left\{L_{i}\right\}_{i \in \mathbb{Z} /(5)}$ of lines in $\mathbb{P}^{4}$ satisfying Conditions $1-3$. Let $\mathfrak{F} \leq \mathfrak{S}$ be the subgroup of order 20 generated by the 5-cycle (01234) and the 4-cycle (1342). Recall the group $G \leq \operatorname{Aut}\left(\Gamma_{\text {Pete }}\right)$ of (2.3) and observe that the mapping (01234) $\mapsto \rho$ and $(1342) \mapsto \tau$ extends to a group isomorphism $f: \mathfrak{F} \stackrel{\sim}{\longrightarrow} G$. Now for $s \in \mathfrak{S}$, we have $\mathcal{A}(s \mathcal{L})=\mathcal{A}(\mathcal{L})$ if and only if $s \in \mathfrak{F}$, in which case the corresponding labelings are related by $\psi_{s \mathcal{L}}=\psi_{\mathcal{L}}(f(s))$.

Of course, labeled Petersen arrangements lead to line arrangements via a reverse construction. Specifically, let $\mathcal{A}$ be a Petersen arrangement and $\psi: \Gamma_{\text {Pete }} \stackrel{\sim}{\longrightarrow} \Gamma(\mathcal{A})$ a labeling. Then we have the indexed collection of lines

$$
\mathcal{L}(\mathcal{A}, \psi)=\left\{\psi(i) \cap \psi\left(i^{\prime}\right)\right\}_{i \in \mathbb{Z} /(5)} .
$$

Remark 4.6. The lines of $\mathcal{L}(\mathcal{A}, \psi)$ correspond to the edges in the perfect matching $\psi\left(E_{5}\right)=\left\{\psi\left(i i^{\prime}\right)\right\}_{i \in \mathbb{Z} /(5)}$ of $\Gamma(\mathcal{A})$. Likewise, for $\alpha \in \operatorname{Aut}\left(\Gamma_{\text {Pete }}\right)$, the lines of $\mathcal{L}(\mathcal{A}, \psi \alpha)$ correspond to the edges in the perfect matching $\psi \alpha\left(E_{5}\right)$ of $\Gamma(\mathcal{A})$. Keeping in mind the notation of Remark 4.5, $\mathcal{L}(\mathcal{A}, \psi \alpha)=\mathcal{L}(\mathcal{A}, \psi)$ as sets if and only if $\alpha \in G=\operatorname{Stab}\left(E_{5}\right) \leq$ $\operatorname{Aut}\left(\Gamma_{\text {Pete }}\right)$, in which case $\mathcal{L}(\mathcal{A}, \psi \alpha)=f^{-1}(\alpha) \mathcal{L}(\mathcal{A}, \psi)$ as indexed sets.

Unfortunately, not every Petersen arrangement can be obtained as in (4.4):

Example 4.7. Let $\mathcal{A}$ be any Petersen arrangement and let $H \subset \mathbb{P}^{4}$ be a general hyperplane. Choose a point $p \notin H$. Then

$$
\mathcal{B}:=\overline{p,(\mathcal{A} \cap H)}
$$

is a Petersen arrangement. Choose a labeling $\psi: \Gamma_{\text {Pete }} \stackrel{\sim}{\longrightarrow} \Gamma(\mathcal{B})$. Then the lines of $\mathcal{L}(\mathcal{B}, \psi)$ all pass through the vertex $p$ and thus do not satisfy Condition 1 . Since $\psi$ was arbitrary, this shows that it is impossible to realize $\mathcal{B}$ as $\mathcal{A}(\mathcal{L})$ for any indexed collection $\mathcal{L}$ of lines.

4.2 LCM Petersen arrangements. Let $\mathcal{L}=\left\{L_{i}\right\}_{i \in \mathbb{Z} /(5)}$ be an indexed collection of lines in $\mathbb{P}^{4}$ satisfying Conditions 1-3, and let $\mathcal{A}=\mathcal{A}(\mathcal{L})$ as in (4.4). While Proposition 4.4 shows that $\mathcal{A}$ is a Petersen arrangement, $\mathcal{A}$ need not be locally Cohen-Macaulay. In light of Proposition 3.6, the study of $1 \mathrm{CM}$ plane arrangements in $\mathbb{P}^{4}$ can be reduced to the study of aCM line arrangements in $\mathbb{P}^{3}$. For our purposes, the following will suffice:

Lemma 4.8. Let $\mathcal{L}$ be an arrangement of lines in $\mathbb{P}^{3}$. If $\Gamma(\mathcal{L})$ is isomorphic to either

(i) the Petersen graph $\Gamma_{\text {Pete }}$,

(ii) the pentagon $C_{5}$, or

(iii) the complete bipartite graph $K_{1,3}$,

then $V_{\mathcal{L}}$ is not arithmetically Cohen-Macaulay.

Proof. In case (i), $V_{\mathcal{L}}$ is a curve of degree 10 and arithmetic genus 6 . In case (ii), $V_{\mathcal{L}}$ is a curve of degree 5 and arithmetic genus 1 . Finally, in case (iii), $V_{\mathcal{L}}$ is a curve of degree 4 and arithmetic genus 0 . In each case, Riemann-Roch now implies that $H^{1}\left(\mathcal{I}_{V_{\mathcal{L}}}(1)\right) \neq 0$. Consequently, $V_{\mathcal{L}}$ is not aCM. 
For instance, the arrangement $\mathcal{B}$ of Example 4.7, being a cone over a non-aCM line arrangement, fails to be $1 \mathrm{CM}$ by Proposition 3.6.

As in the previous section, let $\mathcal{L}=\left\{L_{i}\right\}_{i \in \mathbb{Z} /(5)}$ be an indexed collection of lines in $\mathbb{P}^{4}$ satisfying Conditions $1-3$, and let $\mathcal{A}=\mathcal{A}(\mathcal{L})$ be the resulting Petersen arrangement.

Let $P$ be a plane in $\mathcal{A}$. Then there exist three planes in $\mathcal{A}$ that are adjacent to $P$ in $\Gamma(\mathcal{A})$. Conditions $1-3$ do not imply that the lines along which these planes meet $P$ form a triangle in $P$. Indeed, if these three lines all pass through a single $p \in P$, then $\mathcal{A}_{p}$ fails to be $1 \mathrm{CM}$ at $p$ by case (iii) of Lemma 4.8. Keeping in mind the definition of the "trisecant" lines $M_{i, j, k}$ in Lemma 4.2, we therefore consider

Condition 4. For all $i \in \mathbb{Z} /(5)$, both $M_{i-2, i-1, i} \cap M_{i, i+1, i+2}=\emptyset$ and $M_{i-2, i, i+1} \cap$ $M_{i-1, i, i+2}=\emptyset$.

Notice the similarity of this Condition 4 to Condition 3 via (iv) of Lemma 4.3. Finally, in order to rule out non-lCM points arising as vertices of cones over the line arrangement of case (ii) of Lemma 4.8, we consider:

Condition 5. $\bigcap_{i \in \mathbb{Z} /(5)} H_{i, i+1}=\bigcap_{i \in \mathbb{Z} /(5)} H_{i, i+2}=\emptyset$.

Proposition 4.9. Let $\mathcal{L}$ be an indexed collection of lines in $\mathbb{P}^{4}$ satisfying Conditions 1-5. Then $\mathcal{A}=\mathcal{A}(\mathcal{L})$ is a locally Cohen-Macaulay Petersen arrangement.

Proof. By Corollary 3.10, it suffices to show that $\Gamma\left(\mathcal{A}_{p}\right)$ is connected of order at most 3 for all $p \in V_{\mathcal{A}}$. All cases of this assertion are either obvious (i.e., when $p$ lies in exactly one plane of $\mathcal{A}$ or in exactly two planes of $\mathcal{A}$ that meet in a line) or can be deduced from the

Claim. If $P, Q \in \mathcal{A}$ with $P \cap Q=\{p\}$, then there exists a unique $R \in \mathcal{A}$ meeting both $P$ and $Q$ in lines, in which case $\mathcal{A}_{p}=\{P, Q, R\}$.

Note that $p$ cannot lie in three or more planes meeting pairwise in lines as the Petersen graph contains no 3-cycles. Thus, if $\mathcal{A}_{p}$ consists of three or more planes, there must be at least two of them which meet in a point, in which case the claim applies.

Proof of claim. $P \cap Q=\{p\}$ means that $P$ and $Q$ are not adjacent in $\Gamma(\mathcal{A})$. Since the Petersen graph has no 4-cycles and any two of its vertices lie on a 5-cycle, there is a unique $R \in \mathcal{A}$ meeting both $P$ and $Q$ in lines. These two lines meet at a point of $R$ which, by Remark 3.1 is necessarily $p$, so we are reduced to showing that no other $T \in \mathcal{A}$ contains $p$. Suppose to the contrary, that such a $T$ exists. Then either

(i) $T$ is adjacent to either $P$ or $Q$,

(ii) $T$ is adjacent to none of $P, Q$ and $R$, or

(iii) $T$ and $R$ are adjacent.

In case (i), assume without loss of generality that $P$ and $T$ are adjacent. Then there exists a unique plane $U \in \mathcal{A}$ such that $C:=\Gamma(\{T, P, R, Q, U\})$ is a 5-cycle in $\Gamma(\mathcal{A})$. By Remark 3.1, $p \in U$ as well, so

$$
P \cap R \cap Q \cap U \cap T=\{p\} .
$$


Now either $C=\Gamma\left(\left\{P_{i}: i \in \mathbb{Z} /(5)\right\}\right)$ or $C=\Gamma\left(\left\{P_{i}^{\prime}: i \in \mathbb{Z} /(5)\right\}\right)$ or $C$ contains two of the edges of $E_{5}$. In the first two cases, (4.5) contradicts Condition 5, while in the third, it contradicts Condition 1.

In case (ii), $P$ and $Q$ are distance 2 from $T$, and there exist unique planes $U$ and $U^{\prime} \in \mathcal{A}$ adjacent to $T$ such that $P$ and $Q$ are adjacent to $U$ and $U^{\prime}$ respectively in $\Gamma(\mathcal{A})$. In other words, $C:=\Gamma\left(\left\{T, U, P, R, Q, U^{\prime}\right\}\right)$ is a 6-cycle in $\Gamma(\mathcal{A})$. By repeated applications of Remark 3.1, all planes of $C$ must pass through $p$. Since any 6-cycle in $\Gamma_{\text {Pete }}$ must contain two of the edges of $E_{5}$, this contradicts Condition 1 as in the previous case.

Finally, in case (iii), either $R=P_{i}$ or $R=P_{i}^{\prime}$ for some $i \in \mathbb{Z} /(5)$. If $R=P_{i}$, then without loss of generality, $P=P_{i-1}, Q=P_{i+1}$ and $T=P_{i}^{\prime}$. Then, using (4.3) and (4.1),

$$
\{p\}=P \cap Q \cap R \cap T=M_{i-2, i-1, i} \cap M_{i, i+1, i+2},
$$

contradicting Condition 4. If $R=P_{i}^{\prime}$, an analogous argument again leads to a contradiction to Condition 4.

As it turns out, every locally Cohen-Macaulay Petersen arrangement arises as a result of our construction:

Proposition 4.10. Let $\mathcal{A}$ be a locally Cohen-Macaulay Petersen arrangement and $\psi: \Gamma_{\text {Pete }} \stackrel{\sim}{\longrightarrow} \Gamma(\mathcal{A})$ a labeling. Then $\mathcal{L}(\mathcal{A}, \psi)$ satisfies Conditions $1-5, \mathcal{A}=\mathcal{A}(\mathcal{L}(\mathcal{A}, \psi))$ and $\psi=\psi_{\mathcal{L}(\mathcal{A}, \psi)}$.

Proof. Recall that we set $P_{i}=\psi(i), P_{i}^{\prime}=\psi\left(i^{\prime}\right)$ and $L_{i}=P_{i} \cap P_{i}^{\prime}$ for all $i \in \mathbb{Z} /(5)$ and that $\mathcal{L}=\left\{L_{i}\right\}_{i \in \mathbb{Z} /(5)}$.

For any distinct $i, j \in \mathbb{Z} /(5)$, there is a unique $Q \in \mathcal{A}$ such that $\left\{P_{i}, P_{i}^{\prime}, P_{j}, P_{j}^{\prime}, Q\right\}$ are the vertices of a 5-cycle in $\Gamma(\mathcal{A})$. If $L_{i} \cap L_{j} \neq \emptyset$, then using Remark 3.1, $\left\{P_{i}, P_{i}^{\prime}, P_{j}\right.$, $\left.P_{j}^{\prime}, Q\right\}$ is a cone over a line arrangement with incidence graph isomorphic to the pentagon $C_{5}$. If any other plane of $\mathcal{A}$ also passes through the vertex $p$ of this cone, then, by repeated application of Remark 3.1, all planes of $\mathcal{A}$ have $p$ in common. Thus, either $\mathcal{A}_{p}$ is a cone over a line arrangement with pentagon incidence graph, or it is a cone over a line arrangement with Petersen incidence graph. In either case, $\mathcal{A}$ is not 1CM at $p$ by Lemma 4.8. Thus, $L_{i} \cap L_{j}=\emptyset$, Condition 1 is satisfied and the hyperplanes $H_{i, j}=\overline{L_{i}, L_{j}}$ are defined.

Since $P_{i}$ and $P_{i+1}$ are adjacent in $\Gamma(\mathcal{A})$ for all $i$, they span a hyperplane. This hyperplane must contain $L_{i}$ and $L_{i+1}$ so that

$$
H_{i, i+1}=\overline{P_{i}, P_{i+1}} \quad \text { for all } i \in \mathbb{Z} /(5) .
$$

Similarly,

$$
H_{i, i+2}=\overline{P_{i}^{\prime}, P_{i+2}^{\prime}} \quad \text { for all } i \in \mathbb{Z} /(5) .
$$

Now suppose $\mathcal{L}$ fails Condition 2. By Lemma 4.2, there exist distinct $i, j, k \in \mathbb{Z} /(5)$ such that $L_{i}, L_{j}$, and $L_{k}$ lie in some hyperplane $H$. Without loss of generality, we may assume that either $i=j-1$ and $k=j+1$ or that $i=j-2$ and $k=j+2$. Using (4.6), the first case yields

$$
\overline{P_{j-1}, P_{j}}=H_{j-1, j}=H=H_{j, j+1}=\overline{P_{j}, P_{j+1}} .
$$


Thus, $P_{j-1}$ and $P_{j+1}$ must meet in a line, contradicting their non-adjacency in $\Gamma(\mathcal{A})$. In the second case, a parallel argument using (4.7) shows that $P_{j-2}^{\prime}$ meets $P_{j+2}^{\prime}$ in a line, contradicting their non-adjacency in $\Gamma(\mathcal{A})$. Thus, Condition 2 is satisfied and $P_{i}=$ $H_{i-1, i} \cap H_{i, i+1}$ while $P_{i}^{\prime}=H_{i-2, i} \cap H_{i, i+2}$ for all $i$.

Suppose Condition 3 fails. Then by Lemma 4.3, there exist distinct indices $i, j, k, \ell \in$ $\mathbb{Z} /(5)$ such that $Q=H_{i, j} \cap H_{i, k} \cap H_{i, \ell}$ has dimension 2. Up to obvious reindexing, there are just two cases to consider: either $j=i-1, k=i+1$ and $\ell=i+2$, or $j=i+1$, $k=i+2$ and $\ell=i+3$. In the first of these, $P_{i}=Q \subset H_{i, i+2} \supset P_{i+2}^{\prime}$, contradicting the non-adjacency of $P_{i}$ and $P_{i+2}^{\prime}$. In the second case, $Q=P_{i}^{\prime} \subset H_{i, i+1} \supset P_{i+1}$, contradicting the non-adjacency of $P_{i+1}$ and $P_{i}^{\prime}$. Thus, Condition 3 is satisfied. Note that we have also established that $\mathcal{A}=\mathcal{A}(\mathcal{L}(\mathcal{A}, \psi))$ and $\psi=\psi_{\mathcal{L}(\mathcal{A}, \psi)}$.

Finally, if $\mathcal{L}(\mathcal{A}, \psi)$ fails Conditions 4 or 5 , then $V_{\mathcal{A}}$ contains a point $p$ such that $\mathcal{A}_{p}$ is a cone over a line arrangement with incidence graph isomorphic to $\Gamma_{\text {Pete }}, C_{5}$, or $K_{1,3}$, contradicting, via Lemma 4.8, that $\mathcal{A}$ is $1 \mathrm{CM}$.

4.3 Examples. Conditions 1-5 are non-empty open conditions on the set of indexed collections $\mathcal{L}$ of five lines in $\mathbb{P}^{4}$, so lCM Petersen arrangements exist. To construct a specific example, choose random $2 \times 5$ matrices $A_{i}$ of rank 2 to represent the lines $L_{i} \in \mathcal{L}$ and check by matrix manipulation and rank computations of matrices whether $\mathcal{L}$ satisfies Conditions 1-5.

Alternatively, use a computer algebra system such as MACAULAY2. Represent the lines $L_{i}$ via their ideals, each of which is generated by picking three random linear forms in five variables. In sufficiently large characteristic, Conditions $1-5$ can be verified idealtheoretically. Also, one can check that $\mathcal{A}$ is locally Cohen-Macaulay directly by looking at the second syzygy module of $I_{\mathcal{A}}$; see [1].

Once such a sufficiently generic example has been constructed, one can check computationally that at the corresponding point, the Zariski tangent space to the Hilbert scheme of $\mathbb{P}^{4}$ has dimension 30. Again, see [1]. Now combining Remarks 4.5 and 4.6 and Propositions 4.9 and 4.10, we see that a parameter space for locally Cohen-Macaulay Petersen arrangements is $U / \operatorname{Aut}\left(\Gamma_{\text {Pete }}\right)$ where $U \subset \prod_{\mathbb{Z} /(5)} \mathbb{G}(1,4)$ is the open set of indexed collections of lines satisfying Conditions $1-5$ and Aut $\left(\Gamma_{\text {Pete }}\right)$ acts freely, though only the subgroup $G$ acts by permutation of indices.

Given that $\operatorname{dim} U=30$, we have established:

Theorem 4.11. Let $\mathcal{A}$ be a general locally Cohen-Macaulay Petersen arrangement. Then $V_{\mathcal{A}}$ lies on a unique component of the Hilbert scheme of $\mathbb{P}^{4}$ which is birational to $U / \operatorname{Aut}\left(\Gamma_{\text {Pete }}\right)$. Furthermore, the Hilbert scheme is smooth at $V_{\mathcal{A}}$ of dimension 30 and $V_{\mathcal{A}}$ cannot be smoothed in $\mathbb{P}^{4}$.

Example 4.12. Recall the dihedral subgroup $D=\langle\rho, \sigma\rangle$ of $\operatorname{Aut}\left(\Gamma_{\text {Pete }}\right)$ defined in (2.4). Over $K$, construct a 5 -dimensional representation $V$ of $D$ with basis $\left\{e_{i}\right\}_{i \in \mathbb{Z} /(5)}$, by letting $\rho$ act as the 5-cycle $\left(e_{0} e_{1} e_{2} e_{3} e_{4}\right)$ and $\sigma$ as the product of transpositions $\left(e_{1} e_{4}\right)\left(e_{2} e_{3}\right)$. Then $\sigma$ has eigenvalues 1 and -1 with corresponding eigenspaces $\left\langle e_{0}, e_{1}+e_{4}, e_{2}+e_{3}\right\rangle$, and $\left\langle-e_{1}+e_{4},-e_{2}+e_{3}\right\rangle$, respectively. Consider the $\sigma$-invariant two-dimensional subspace $W$ of $V$ spanned by $v_{1}=e_{0}+e_{2}+e_{3}$ and $v_{2}=-e_{1}-e_{2}+e_{3}+e_{4}$. 
Now in the induced projective representation $\mathbb{P}(V)$, the line $L_{0}:=\mathbb{P}(W)$ is also $\sigma$ invariant. For $i \in \mathbb{Z} /(5)$, set $L_{i}:=\rho^{i}\left(L_{0}\right)$. Then as a set, $\mathcal{L}=\left\{L_{i}\right\}_{i \in \mathbb{Z} /(5)}$ is invariant under the action of $D \hookrightarrow \operatorname{PGL}(V)$, and it is straightforward to show that $\mathcal{L}$ satisfies Conditions $1-5$. Thus $\mathcal{A}(\mathcal{L})$ is a locally Cohen-Macaulay Petersen arrangement (see [1] for a direct verification), invariant under the action of $D$. Note, however, that the corresponding labelings change in the obvious way: $\psi_{\delta \mathcal{L}}=\psi_{\mathcal{L}} \circ \delta$ for $\delta \in D$.

\section{Generators of $I_{\mathcal{A}}$}

Let $\mathcal{A}$ be a locally Cohen-Macaulay Petersen arrangement and fix a labeling $\psi: \Gamma_{\text {Pete }} \rightarrow$ $\Gamma(A)$. As before, let $P_{i}=\psi(i)$ and $P_{i}^{\prime}=\psi\left(i^{\prime}\right)$ for $i \in \mathbb{Z} /(5)$. The ideal $I_{\mathcal{A}}$ is obtained as the intersection of the ideals of the planes in $\mathcal{A}$ and, by means of an example, one can determine that a minimal set of generators for $I_{\mathcal{A}}$ consists of six quintics and five sextics. The main goal of this section is to provide a combinatorial description of these generators. Among other things, this description will allow us to show that there are exactly five 6secant lines to $V_{\mathcal{A}}$.

Let $u$ and $v$ be adjacent vertices of $\Gamma_{\text {Pete }}$ and $e=u v$ the corresponding edge. Then the planes $\psi(u), \psi(v) \in \mathcal{A}$ span a hyperplane which we denote

$$
H^{u, v}=H^{e}:=\overline{\psi(u), \psi(v)} .
$$

For each of the six perfect matchings $E_{i}$ of $\Gamma_{\text {Pete }}$ (see Section 2), we define a quintic hypersurface in $\mathbb{P}^{4}$ by

$$
Q_{i}:=\bigcup_{e \in E_{i}} H^{e}
$$

Let $q_{i} \in S$ be a quintic polynomial that defines $Q_{i}$. We will prove that $q_{i} \in\left(I_{\mathcal{A}}\right)_{5}$. For each $i \in \mathbb{Z} /(5)$, define

$$
l_{i}:=H^{i, i^{\prime}} \cap H^{(i+1)^{\prime},(i-1)^{\prime}} \cap H^{(i+2),(i-2)} .
$$

Lemma 5.1. The subspaces $l_{i}$ satisfy:

(i) $l_{i}$ is a line.

(ii) $l_{i} \not \subset V_{\mathcal{A}}$.

(iii) The $l_{i}$ are pairwise disjoint.

Proof. The techniques are similar and rely on knowing that $\mathcal{A}$ is 1CM and Petersen. For (i), without loss of generality consider the case $i=0$. The three hyperplanes $H^{0,0^{\prime}}, H^{1^{\prime}, 4^{\prime}}$ and $H^{2,3}$ are distinct. (If, e.g., $H^{0,0^{\prime}}=H^{2,3}$, then $P_{0}$ and $P_{2}$ meet in a line, contradicting that $\mathcal{A}$ is Petersen.) So if $l_{0}$ is not a line, it is a plane. Suppose that were the case. Consider the three points $P_{0} \cap P_{1}^{\prime}, P_{1}^{\prime} \cap P_{2}$ and $P_{2} \cap P_{0}$; by Remark 3.1, all three must lie in $P_{1}$. Since $\mathcal{A}$ is $1 \mathrm{CM}$, they cannot coincide. (See case (iii) of Lemma 4.8.) They cannot be collinear since no two of $P_{1}^{\prime}, P_{2}$, and $P_{0}$ meet in a line. Thus, they span $P_{1}$. Since each of them lies in two of the hyperplanes defining $l_{0}$, they also span $l_{0}$ and we conclude $P_{1}=l_{0}$. But this would give, e.g., $\operatorname{dim} P_{1} \cap P_{0}^{\prime}=1$, again contradicting that $\mathcal{A}$ is Petersen. 
To prove (ii), suppose to the contrary, for example, that $l_{0} \subset P_{0}$. (The remaining cases are similar.) Then the four points $P_{0} \cap P_{1}^{\prime}, P_{0} \cap P_{2}, P_{0} \cap P_{4}^{\prime}$ and $P_{0} \cap P_{3}$ lie on $l_{0}$, and they must be distinct since $\mathcal{A}$ is $1 \mathrm{CM}$. Now by Remark 3.1, the first two of these lie on $P_{1}$ and we deduce $l_{0}=P_{0} \cap P_{1}$. Working with the third and fourth points, we see that $l_{0}=P_{0} \cap P_{4}$ as well, contradicting that $\operatorname{dim} P_{1} \cap P_{4}$ consists of a point.

Finally, to prove (iii), suppose, for example, that $l_{i}$ and $l_{i+1}$ share a point. Then $P_{i}^{\prime}$, $P_{i+1}^{\prime}$ and $P_{i-2}$ must intersect, which, as we saw in Section 4.2, contradicts the assumption that $\mathcal{A}$ is an ICM Petersen arrangement. The remaining cases follow in a similar manner.

Finally, suppose that the arrangement $\mathcal{A}$ satisfies the following open condition:

Condition 6. For each perfect matching $E_{i}$ of $\Gamma_{\text {Pete }}, \bigcap_{e \in E_{i}} H^{e}=\emptyset$.

Then for each $i \in \mathbb{Z} /(5)$,

$$
p_{i}:=\bigcap_{e \in E_{i} \backslash E_{5}} H^{e},
$$

is a point and we have the following lemma:

Lemma 5.2. Suppose that $\mathcal{A}$ satisfies Condition 6. Then the union of $V_{\mathcal{A}}$, the five lines $\left\{l_{i}\right\}$ and the five points $\left\{p_{i}\right\}$ is cut out set theoretically by the five quintic hypersurfaces $Q_{0}, \ldots, Q_{4}$.

Proof. Let $Q=Q_{0} \cap \cdots \cap Q_{4}$ denote the intersection of the five quintic hypersurfaces and let $I_{Q}=\left(q_{0}, \ldots, q_{4}\right)$ denote the ideal generated by the five quintics. Then a point $p$ of $\mathbb{P}^{4}$ lies in $Q$ if and only if for each $i \in \mathbb{Z} /(5)$, there exists at least one $e \in E_{i}$ such that $H^{e}$ contains $p$. A point $p$ of $Q$ lies in $V_{\mathcal{A}}$ if and only if for two distinct elements $i$ and $j$ of $\mathbb{Z} /(5)$, there exist $e \in E_{i}$ and $f \in E_{j}$ such that $H^{e}$ and $H^{f}$ contain $p$ and intersect in one of the planes in $\mathcal{A}$. (The latter condition is equivalent to the condition that the edges $e$ and $f$ share a common vertex.)

Thus, a point $p$ lies in the closure of the complement of $V_{\mathcal{A}}$ in $Q$ (whose ideal is the ideal quotient $I_{Q}: I_{\mathcal{A}}$ ) if $p \in \bigcap_{e \in E} H^{e}$ for some matching $E$ (not necessarily perfect) of $\Gamma_{\text {Pete }}$ which is minimal among matchings $E$ such that $E \cap E_{i} \neq \emptyset$ for all $i \in \mathbb{Z} /(5)$. Any such $E$ is either $E_{i} \backslash E_{5},\left\{i i^{\prime},(i+1)^{\prime}(i-1)^{\prime},(i+2)^{\prime}(i-2)^{\prime}\right\}$, or $E_{5}$. However, by Condition 6 , the last subset cannot occur. Thus, the residual variety consists precisely of the union of the $p_{i}$ and the $l_{i}$, which completes the proof.

Theorem 5.3. The union of $V_{\mathcal{A}}$ and the five lines $\left\{l_{i}\right\}$ is scheme-theoretically cut out by the six quintic hypersurfaces $Q_{0}, \ldots, Q_{5}$.

Proof. For the set-theoretic statement, it is enough, by Lemma 5.2, to show that if $i \in$ $\mathbb{Z} /(5)$ and $p_{i} \notin V_{\mathcal{A}}$, then $p_{i} \notin H^{e}$ for all $e \in E_{5}$. Condition 6 implies that $H^{i, i^{\prime}}$ does not contain $p_{i}$. On the other hand, if $j \neq i$, then either $(j)(j+1) \in E_{i}$ or $(j-1)(j) \in E_{i}$, so that $p_{i} \in H^{j, j^{\prime}}$ would imply that $p_{i} \in P_{j}=H^{j, j^{\prime}} \cap H^{j-1, j}=H^{j, j^{\prime}} \cap H^{j, j+1}$, contradicting that $p_{i} \notin V_{\mathcal{A}}$. A computation with MACAULAY2 shows that the union of $V_{\mathcal{A}}$ 
and the five lines $\left\{l_{i}\right\}$ is not only set-theoretically but also scheme-theoretically cut out by the six quintic hypersurfaces $Q_{0}, \ldots, Q_{5}$. Indeed, the degree 5 component of the ideal is the span of the corresponding quintic forms $q_{0}, \ldots, q_{5}$. Since this is an open condition, the vanishing ideal of the union of a general $1 \mathrm{CM}$ Petersen arrangement and the five lines $\left\{l_{i}\right\}$ is minimally generated by the quintics $q_{0}, \ldots, q_{5}$.

As a corollary, we have:

Corollary 5.4. Let $V_{\mathcal{A}}$ be a general lCM Petersen arrangement. Then there are exactly five 6-secant lines to $V_{\mathcal{A}}$.

Proof. Let $l$ be a 6-secant line to $V_{\mathcal{A}}$. Then any quintic hypersurface containing $V_{\mathcal{A}}$ contains $l$. It follows from Theorem 5.3 that $l=l_{i}$ for some $i \in \mathbb{Z} /(5)$. But for each $i \in \mathbb{Z} /(5)$, the line $l_{i}$ meets the six planes $P_{i}, P_{i^{\prime}}, P_{(i+1)^{\prime}}, P_{(i-1)^{\prime}}, P_{i+2}$ and $P_{i-2}$, but does not meet the lines $P_{i} \cap P_{i^{\prime}}, P_{(i+1)^{\prime}} \cap P_{(i-1)^{\prime}}$ and $P_{i+2} \cap P_{i-2}$ and hence does not lie in the planes it meets. So $l_{i}$ is a 6-secant to $V_{\mathcal{A}}$.

Remark 5.5. The general ICM Petersen arrangement satisfies Condition 6. One can verify, however, that the arrangement of Example 4.12 does not. In that case, the five quintics $q_{0}, \ldots, q_{4}$ are linearly independent, but $q_{5}$ can be written as a linear combination of $q_{0}, \ldots, q_{4}$. We will discuss this example further in the last section.

We recall a well-known fact:

Lemma 5.6. If four 2-planes in $\mathbb{P}^{4}$ meet pairwise in distinct points, then there exists a unique cubic hypersurface containing them.

Now for each $i \in \mathbb{Z} /(5)$, let $C_{i}$ be the unique cubic hypersurface in $\mathbb{P}^{4}$ containing the four planes $P_{i+1}, P_{(i+2)^{\prime}}, P_{(i-2)^{\prime}}, P_{i-1}$ (which correspond to the maximal independent vertex set $V_{i}$ defined in Section 2) and set

$$
S_{i}:=H^{i, i^{\prime}} \cup H^{(i+1)^{\prime},(i-1)^{\prime}} \cup H^{(i+2),(i-2)} \cup C_{i} .
$$

Observe that $V_{\mathcal{A}} \cup l_{i} \subset S_{i}$.

Theorem 5.7. $V_{\mathcal{A}}$ is scheme-theoretically cut out by the six quintic hypersurfaces $Q_{0}$, $\ldots, Q_{5}$ and any two different sextic hypersurfaces $S_{j}$ and $S_{k}$.

Proof. Since the $l_{j}$ are pairwise skew, it suffices to show that $V_{\mathcal{A}} \cup l_{j}$ is scheme-theoretically cut out by $Q_{0}, \ldots, Q_{5}$ and $S_{j}$ for every $j \in \mathbb{Z} /(5)$. Without loss of generality, we may assume that $j=0$. Let $i \neq 0$. If $l_{i}$ does not lie in $S_{0}$, then, since $l_{i}$ is a 6-secant to $V_{\mathcal{A}}$,

$$
\left(l_{i} \cap S_{0}\right) \subset V_{\mathcal{A}}
$$

and we are done by Theorem 5.3. Thus, our task is to prove that $l_{i}$ does not lie in $S_{0}$.

Here we will only show that $l_{1}$ does not lie in $S_{0}$; essentially the same proof applies to the remaining cases. Suppose, to the contrary, that $l_{1} \subset S_{0}$. Then $l_{1}$ lies in $C_{0}$ or in one of the hyperplanes $H^{0,0^{\prime}}, H^{1^{\prime}, 4^{\prime}}$ or $H^{2,3}$. Given that $l_{1}=H^{1,1^{\prime}} \cap H^{2^{\prime}, 0^{\prime}} \cap H^{3,4}$, we deduce 
that $l_{1}$ lies in either $P_{0}^{\prime}=H^{0,0^{\prime}} \cap H^{0^{\prime}, 2^{\prime}}, P_{1^{\prime}}=H^{1,1^{\prime}} \cap H^{1^{\prime}, 4^{\prime}}$ or $P_{3}=H^{2,3} \cap H^{3,4}$, contradicting Lemma 5.1.

Finally, suppose that $l_{1} \subset C_{0}$. Recall that $l_{1}$ meets (but is not contained in) $P_{1}, P_{4}$ and $P_{2}^{\prime}$. Since $C_{0}$ contains these three planes, $C_{0}$ must be the union of the three distinct hyperplanes spanned by $l_{1}$ and these planes. Thus the fourth plane, $P_{3}^{\prime}$ that $C_{0}$ contains must lie in one of these hyperplanes and therefore meets either $P_{1}, P_{4}$, or $P_{2}^{\prime}$ in a line, contradicting that $\mathcal{A}$ is Petersen.

Remark 5.8. Let $\mathcal{A}$ be a Petersen arrangement and let $I_{\mathcal{A}}$ be its vanishing ideal. One can show that $I_{\mathcal{A}}$ is pl-generated, i.e., that it is generated by products of linear forms. Recall that every quintic polynomial $q_{i}$ we defined is a product of linear forms. Furthermore, five partitions of $E\left(\Gamma_{\text {Pete }}\right)$ arise naturally from the 6-cycles of $\Gamma_{\text {Pete. }}$. To each of these set partitions, we can attach a sextic polynomial which is a product of linear forms. One then shows that these quintics and sextics together generate the same ideal as the $q_{i}$ and the forms vanishing on the $S_{j}$. Arrangements defined by products of linear forms have been studied by Björner, Peeva and Sidman [2].

\section{Smooth general-type surfaces of degree 15}

Once again, let $\mathcal{A}=\left\{P_{i}, P_{i}^{\prime}\right\}_{i \in \mathbb{Z}_{5}}$ be a (labeled) lCM Petersen arrangement. We have shown that the vector space formed by quintic hypersurfaces containing $V_{\mathcal{A}}$ has dimension 6, so $V_{\mathcal{A}}$ can be linked in the complete intersection of two quintic hypersurfaces to a surface of degree 15 . In this section, we will study the general surface arising in this way. First, however, we recall (from [8] or [7], for example) the main definition and some basic results of liaison theory.

Definition 6.1. Let $X$ and $X^{\prime}$ be surfaces in $\mathbb{P}^{4}$ with no irreducible component in common. Then $X$ and $X^{\prime}$ are said to be linked by a complete intersection of type $(m, n)$ if there exist hypersurfaces $Y$ and $Y^{\prime}$ of degrees $m$ and $n$ respectively such that $Y \cap Y^{\prime}=$ $X \cup X^{\prime}$ scheme-theoretically.

In this situation, $X$ is $1 \mathrm{CM}$ if and only if $X^{\prime}$ is $1 \mathrm{CM}, X \cap X^{\prime}$ is a curve, and there are two standard exact sequences of linkage,

$$
0 \longrightarrow \omega_{X} \longrightarrow \mathcal{O}_{X \cup X^{\prime}}(m+n-5) \longrightarrow \mathcal{O}_{X^{\prime}}(m+n-5) \longrightarrow 0
$$

and

$$
0 \longrightarrow \omega_{X} \longrightarrow \mathcal{O}_{X}(m+n-5) \longrightarrow \mathcal{O}_{X \cap X^{\prime}}(m+n-5) \longrightarrow 0 .
$$

The first sequence yields the relation between the Euler-Poincaré characteristics:

$$
\chi\left(\mathcal{O}_{X^{\prime}}\right)=\chi\left(\mathcal{O}_{X \cup X^{\prime}}\right)-\chi\left(\omega_{X}(5-m-n)\right) .
$$

Letting $d$ and $d^{\prime}$ denote the degrees of $X$ and $X^{\prime}$ and $\pi$ and $\pi^{\prime}$ their sectional genera, the corresponding sequence for linkage of curves in $\mathbb{P}^{3}$ yields the relation

$$
\pi-\pi^{\prime}=\frac{1}{2}(m+n-4)\left(d-d^{\prime}\right) .
$$


Theorem 6.2. Let $V_{\mathcal{A}}$ be a general lCM Petersen arrangement. Then the surface $X$ linked to $V_{\mathcal{A}}$ by two general quintic hypersurfaces is smooth, non-minimal and of general type, with degree $d=15$, sectional genus $\pi=21$, Euler-Poincaré characteristic $\chi=\chi\left(\mathcal{O}_{X}\right)=5$, geometric genus $p_{g}=4$, irregularity $q=0$, and Euler number $e(X)=55$. Moreover, $X$ is embedded in $\mathbb{P}^{4}$ by the linear system

$$
\left|H_{\min }-\sum_{i=0}^{4} l_{i}\right|,
$$

where $H_{\min }$ is the hyperplane class of the minimal model of $X$ and the $l_{i}$ are the lines of (5.1).

Proof. As we have shown in Section $5,\left(I_{\mathcal{A}}\right)_{5}$ is generated by six quintics. Thus, $V_{\mathcal{A}}$ is linked - via a complete intersection of two general elements of $\left(I_{\mathcal{A}}\right)_{5}$ - to a surface $X$ of degree 15. Furthermore, a computation with MACAULAY2 (see [1]) shows that $X$ is smooth for a particular choice of $\mathcal{A}$ and quintics in $I_{\mathcal{A}}$. Hence $X$ is smooth in general.

By (6.2), $X$ has sectional genus $\pi=21$ and by (6.1), $X$ has Euler-Poincaré characteristic $\chi=5$.

Let $H$ and $K$ be a hyperplane section and a canonical divisor of $X$ respectively. Recall the double point formula for surfaces in $\mathbb{P}^{4}[5]$ :

$$
d^{2}-10 d-5 H . K-2 K^{2}+12 \chi=0 .
$$

Using this formula, it follows that $K^{2}=5$, showing that $X$ is of general type. This also proves that $e(X)=55$, because $\chi=\frac{1}{12}\left(K^{2}+e\right)$. A computation with MACAULAY2 shows that $p_{g}=4$, and hence $q=\chi-p_{g}-1=0$.

Recall that the lines $l_{0}, \ldots, l_{4}$ of $(5.1)$ are 6 -secants to $V_{\mathcal{A}}$. From the first standard linkage sequence, we obtain:

$$
0 \longrightarrow \mathcal{I}_{X \cup V_{\mathcal{A}}}(5) \longrightarrow \mathcal{I}_{\mathcal{A}}(5) \longrightarrow \mathcal{O}_{X}(K) \longrightarrow 0 .
$$

Since $X \cup V_{\mathcal{A}}$ is a complete intersection of two quintic hypersurfaces and since $\mathcal{I}_{\mathcal{A}}(5)$ is globally generated by its sections outside $\bigcup l_{i}$, the canonical bundle $\omega_{X}$ is also globally generated outside $\bigcup l_{i}$. As a consequence, $\bigcup l_{i}$ is the fixed part of the canonical linear series, and $l_{0}, \ldots, l_{4}$ are the exceptional lines of $X$. In particular, the embedding linear system of $X$ has the desired form.

Remark 6.3. Let $\varphi_{|H+K|}: X \rightarrow \mathbb{P}^{N}$ be the adjunction map, where $N=\pi+p_{a}-1=$ $21+4-1=24$. Then the minimal model $X_{\min }$ of $X$ is obtained as the image of $X$ under $\varphi_{|H+K|}$. Let $H_{\min }$ be its hyperplane class and let $K_{\min }$ be its canonical divisor. Then $X_{\min }$ has degree $H_{\min }^{2}=(H+K)^{2}=70$. Since $H_{\min } \cdot K_{\min }=(H+K) \cdot K=30$, the sectional genus of $X_{\min }$ is 51 . We note that the canonical model of $X_{\min }$ is a singular surface of degree 10 in $\mathbb{P}^{3}$.

Remark 6.4. For a general surface $X$ obtained in this way, $H^{0}\left(\mathcal{I}_{X}(5)\right)=2$. Therefore, since the family of $1 \mathrm{CM}$ Petersen arrangements has dimension 30 and since the space 
of quintics containing each Petersen arrangement has dimension 6, the family of smooth general type surfaces of degree 15 obtained from the Petersen arrangements via linkage has dimension $38=30+2(6-2)$. On the other hand, a standard computation shows that $\chi\left(N_{X / \mathbb{P}^{4}}\right)=35$. We conclude that $\operatorname{dim} H^{1}\left(N_{X / \mathbb{P}^{4}}\right) \geq 3$, but at present, we are unable to show that equality holds in general. Consequently, we cannot rule out the possibility that the Hilbert scheme of $\mathbb{P}^{4}$ may be obstructed at the general surface $X$ obtained via our construction.

Now let $P \in \mathcal{A}$. Then exactly three distinct planes of $\mathcal{A}$ meet $P$ in lines, and since $\mathcal{A}$ is 1CM, these three lines meet pairwise in three distinct points $r_{1}, r_{2}$, and $r_{3}$ of $P$.

Lemma 6.5. There exists a quintic plane curve $Q \subset P$, not passing through $r_{1}, r_{2}$ or $r_{3}$, such that $X \cap P=Q \cup\left\{r_{1}, r_{2}, r_{3}\right\}$ scheme-theoretically.

Proof. Set $\mathcal{B}=\mathcal{A} \backslash\{P\}$ and let $U=X \cup V_{\mathcal{B}}$. Then $P$ and $U$ are linked (by the same complete intersection of degree $(5,5)$ that links $V_{\mathcal{A}}$ and $X$ ), so $C=P \cap U$ is a curve and the second standard exact sequence of linkage becomes

$$
0 \longrightarrow \omega_{P} \longrightarrow \mathcal{O}_{P}(5) \longrightarrow \mathcal{O}_{C}(5) \longrightarrow 0 .
$$

Consider the divisorial exact sequence:

$$
0 \longrightarrow \mathcal{O}_{P}(-C) \longrightarrow \mathcal{O}_{P} \longrightarrow \mathcal{O}_{C} \longrightarrow 0 .
$$

Let $L \subset P$ be a line so that $\omega_{P}=\mathcal{O}_{P}(-3 L)$. By comparing (6.3) with (6.4) twisted by $\mathcal{O}_{P}(5)$, we conclude that $C \equiv 8 L$ in $P$. Now there are exactly three planes of $\mathcal{B}$ that intersect $P$ in lines, so the intersection $X \cap P$ contains a curve $Q \equiv 8 L-3 L=5 L$, i.e., a plane curve of degree 5 .

Now let $K=K_{X}$ be a canonical divisor for $X$. Let $X \cdot P$ denote the intersection product of $X$ and $P$. Recall that the equivalence of $Q$ for the intersection product $X \cdot P$ in $\mathbb{P}^{4}$ is given by a formula of Todd:

$$
(X \cdot P)^{Q}=(K \cdot Q)_{X}+\left(K_{P} \cdot Q\right)_{P}-\left(K_{\mathbb{P}^{4}} \cdot Q\right)_{\mathbb{P}^{4}}-K_{Q} ;
$$

see [3, Example 9.1.7]. Since $Q$ is a quintic plane curve, $K_{P} \cdot Q=-15, K_{\mathbb{P}^{4}} \cdot Q=-25$, and $\operatorname{deg} K_{Q}=2 \cdot 6-2=10$. The length of the residual scheme is

$$
\begin{aligned}
\operatorname{deg}(X \cdot P)-\operatorname{deg}(X \cdot P)^{Q} & =\operatorname{deg} X-(K . Q)+15-25+10 \\
& =15-K \cdot Q .
\end{aligned}
$$

Consider the exact sequence of linkage:

$$
0 \longrightarrow \omega_{X} \longrightarrow \mathcal{O}_{X}(5) \longrightarrow \mathcal{O}_{D}(5) \longrightarrow 0,
$$

where $D=X \cap V_{\mathcal{A}}$. By comparing this sequence with

$$
0 \longrightarrow \mathcal{O}_{X}(-D) \longrightarrow \mathcal{O}_{X} \longrightarrow \mathcal{O}_{D} \longrightarrow 0,
$$


we deduce that $D \equiv 5 H-K$ in $X$ and therefore

$$
K . D=K .(5 H-K)=120 .
$$

On the other hand, $C$ is the sum of 10 quintics, one in each plane of $\mathcal{A}$, and we deduce that $K . Q=120 / 10=12$. So by (6.5), the residual scheme to $Q$ in $P \cap X$ has length $15-12=3$.

Now $V_{\mathcal{B}}$ fails to be 1CM precisely at $r_{1}, r_{2}$ and $r_{3}$ and since $V_{\mathcal{B}}$ and $X \cup P$ are linked (by the same pair of quintics that link $X$ to $V_{\mathcal{A}}$ ), $X \cup P$ fails to be lCM precisely at $r_{1}, r_{2}$, $r_{3}$. It follows that the residual scheme to $Q$ in $P \cap X$ is supported at the $r_{i}$, completing the proof.

Theorem 6.6. The surface $X$ admits a two-dimensional family of 5-secant lines, a onedimensional family of 6-secant lines, and fifteen 7-secant lines.

Proof. Keeping the notation, we see from Lemma 6.5 that any line in $P \in \mathcal{A}$ meets $Q$-and hence $X$-in five points (possibly with multiplicity). Furthermore, any line in $P$ passing through $r_{1}, r_{2}$, or $r_{3}$ meets $X$ in that point in addition to the five points where it meets $Q$. Finally, any of the three lines $\overline{r_{i}, r_{j}}$ meets $X$ in those two points in addition to its five points of intersection with $Q$. Since there are three such lines in each of the ten planes $P$ of $\mathcal{A}$ and since each such line consists of the intersection of two of those planes, we arrive at the count of fifteen 7 -secants.

\section{A general-type surface with symmetries}

Let $\mathcal{A}$ be the plane arrangement constructed in Example 4.12. Recall that $\mathcal{A}$, and thus $V_{\mathcal{A}}$ has $D=\left\langle\rho, \sigma: \rho^{5}=\sigma^{2}=e, \rho \sigma=\sigma \rho^{4}\right\rangle$ as a group of symmetries.

Consider the perfect matching $E_{5}=\left\{i i^{\prime}\right\}_{i \in \mathbb{Z} /(5)} \subset E\left(\Gamma_{\text {Pete }}\right)$. Recall that each of the other perfect matchings are obtained as

$$
E_{j}=\rho^{j} \tau E_{5}
$$

for $j \in \mathbb{Z} /(5)$. In Section 5, we attached to each $E_{j}$ a quintic polynomial $q_{j} \in I_{\mathcal{A}}$. It is easy to check that the first five quintic polynomials, $q_{0}, \ldots, q_{4}$ are linearly independent. Let $W$ be the vector space they span; it is clear that $D$ acts on $W$.

We now denote by $\zeta$ a nontrivial 5th root of unity. Let $w_{1}$ and $w_{2}$ be eigenvectors for the action of $\rho$ with eigenvalues $\zeta$ and $\zeta^{2}$ respectively. Then, for each $i \in\{1,2\}$, the quintics $w_{i}$ and $\sigma\left(w_{i}\right)$ span a two-dimensional subspace $W_{i}$ of $W$ which is invariant under $D$. Take two general quintic polynomials in either $W_{1}$ or $W_{2}$. Then the surface $X$ linked, via these two quintics, to $V_{\mathcal{A}}$ is smooth. Thus, $X$ is a smooth $D$-invariant general type surface in $\mathbb{P}^{4}$.

As in Example 4.12, let $V$ be the underlying vector space of $\mathbb{P}^{4}$ with basis $\left\{e_{i}\right\}_{i \in \mathbb{Z} /(5)}$. The action of $D$ is given by $\rho\left(e_{i}\right)=e_{i+1}$ and $\sigma\left(e_{i}\right)=e_{5-i}$. In other words, $V$ is the standard permutation representation of $D$. Recall that $V$ contains the trivial representation of $D$, which is spanned by the vector $\sum_{i \in \mathbb{Z} /(5)} e_{i}$. Let $p$ be the corresponding point of 
$\mathbb{P}^{4}$. We can check that each of the quintic hypersurfaces used to link $V_{\mathcal{A}}$ to $X$ contains this point. Thus $p$ lies in the complete intersection $X \cup V_{\mathcal{A}}$. The point $p$ cannot lie in any plane in $\mathcal{A}$ since if $p$ lies in $P \in \mathcal{A}$, then $\rho^{i}(P)$ would also contain $p$, which contradicts our assumption. Therefore $p \in X$. Thus, we have proved:

Theorem 7.1. There exists a smooth surface $X \subset \mathbb{P}^{4}$ as in Theorem 6.2 and Theorem 6.6, admitting an action of the dihedral group on five elements which is free except at the single fixed point $p \in X$.

\section{References}

[1] H. Abo, H. P. Kley, C. Peterson. http://www.uidaho.edu/ abo/programs.html

[2] A. Björner, I. Peeva, J. Sidman, Subspace arrangements defined by products of linear forms. $J$. London Math. Soc. (2) 71 (2005), 273-288. MR2122428 (2006d:05194) Zbl 1111.13018

[3] W. Fulton, Intersection theory. Springer 1984. MR732620 (85k:14004) Zbl 0541.14005

[4] D. Grayson, M. Stillman, Macaulay 2, a software system for research in algebraic geometry. http://www.math.uiuc.edu/Macaulay2/

[5] R. Hartshorne, Algebraic geometry. Springer 1977. MR0463157 (57 \#3116) Zbl 0367.14001

[6] C. Huneke, B. Ulrich, General hyperplane sections of algebraic varieties. J. Algebraic Geom. 2 (1993), 487-505. MR1211996 (94b:14046) Zbl 0808.14041

[7] J. C. Migliore, Introduction to liaison theory and deficiency modules. Birkhäuser 1998. MR1712469 (2000g:14058) Zbl 0921.14033

[8] C. Peskine, L. Szpiro, Liaison des variétés algébriques. I. Invent. Math. 26 (1974), 271-302. MR0364271 (51 \#526) Zbl 0298.14022

Received 20 June, 2007

H. Abo, Department of Mathematics, University of Idaho, Moscow, ID 83844, USA http://www.webpages.uidaho.edu/ abo Email: abo@uidaho.edu

H. Kley, Department of Mathematics, Colorado State University, Fort Collins, CO 80523, USA http://www.math.colostate.edu/ kley Email: kley@math.colostate.edu

C. Peterson, Department of Mathematics, Colorado State University, Fort Collins, CO 80523, USA http://www.math.colostate.edu/ peterson Email: peterson@math.colostate.edu 